\title{
artigo
}

Nascimento, C.G.; Taveres, M.G.V.; Nunes, M.S.; Alves, L.L.; Barreto, M.S.B.; Santos, I.I.; Menezes, L.R.O.; Oliveira, R.I.S.;

O uso de toxina botulínica no tratamento de rugas dinâmicas

\section{0 uso de toxina botulínica no tratamento de rugas dinâmicas}

The use of botulinum toxin in the treatment of dynamic wrinkles

El uso de la toxina botulínica em el tratamento de las rugas dinámicas

\section{RESUMO}

O objetivo deste artigo foi analisar a eficácia da Toxina Botulínica tipo A no tratamento de rugas. Este tratamento é utilizado visando retardar o envelhecimento da pele. Para tanto, o estudo da ação da Toxina Botulínica e conhecimento à aplicação do "Botox", nome popularmente conhecido, no tratamento de rugas dinâmicas são fundamentais, de forma a evitar a ocorrência de possíveis complicações no uso. O método aplicado foi uma revisão de literatura, sendo a pesquisa realizada pelos bancos de dados SciELO, PubMed, Science Direct, MedLine e Bireme. Foi possível identificar nos resultados dos estudos científicos, a eficácia da Toxina, bem como reafirmar a satisfação dos pacientes que utilizam este tratamento. Porém, conclui-se que, ainda é preciso novos estudos para o avanço deste procedimento estético para contribuir na ampliação do quadro de atuação do Biomédico e viabilizar recursos para o tratamento de pacientes submetidos ao Botox.

DESCRITORES: Toxina Botulínica; Envelhecimento; Botox; Rugas.

\section{ABSTRACT}

The purpose of this article was to analyze the efficacy of Botulinum Toxin type $A$ in the treatment of wrinkles that is used to delay skin aging. Therefore, the study of the action of Botulinum Toxin and knowledge of the application of "Botox", a popular name, in the treatment of dynamic wrinkles are essential, in order to avoid the occurrence of possible complications in use. The method applied was a literature review, and the research was carried out by the SciELO, PubMed, Science Direct, MedLine and Bireme databases. It was possible to identify the results of scientific studies, the effectiveness of Toxin, as well as reaffirm the satisfaction of patients who use this treatment. However, it is concluded that, further studies are needed to advance this aesthetic procedure to contribute to the expansion of the Biomedical field of action and enable resources for the treatment of patients undergoing Botox.

DESCRIPTORS: Botulinum Toxin; Aging; Botox; Wrinkles.

\section{RESUMEN}

El propósito de este artículo fue analizar la eficacia de la Toxina Botulínica tipo A en el tratamiento de arrugas que se utiliza para retrasar el envejecimiento cutáneo. Por tanto, el estudio de la acción de la Toxina Botulínica y el conocimiento de la aplicación de "Botox", un nombre popular, en el tratamiento de las arrugas dinámicas son fundamentales, para evitar la aparición de posibles complicaciones en su uso. El método aplicado fue una revisión de la literatura, y la investigación fue realizada por las bases de datos SciELO, PubMed, Science Direct, MedLine y Bireme. Fue posible identificar los resultados de estudios científicos, la efectividad de la Toxina, así como reafirmar la satisfacción de los pacientes que utilizan este tratamiento. Sin embargo, se concluye que se necesitan más estudios para avanzar en este procedimiento estético para contribuir a la expansión del campo de acción Biomédico y habilitar recursos para el tratamiento de los pacientes sometidos a Botox.

DESCRIPTORES: Toxina Botulínica; Envejecimiento; Botox; Arrugas.

RECEBIDO EM: 30/10/2020 APROVADO EM: 09/11/2020

\section{Crisabete Gomes do Nascimento}

Graduando(a) em Biomedicina, Centro Universitário Estácio de Sergipe, Aracaju, Brasil.

ORCID: 0000-0002-9230-3804 


\section{Marcos Garcia Valois Tavares}

Graduando(a) em Biomedicina, Centro Universitário Estácio de Sergipe, Aracaju, Brasil.

ORCID: 0000-0001-9143-6632

\section{Mylena dos Santos Nunes}

Graduando(a) em Biomedicina, Centro Universitário Estácio de Sergipe, Aracaju, Brasil.

ORCID: 0000-0002-3464-044X

\section{Lumar Lucena Alves}

Medical Technologist, Gerald Champion Regional Medical Center - GCRMC, NM, USA, Graduado em Biomedicina, Universidade Tiradentes, Aracaju, Brasil.

ORCID: 0000-0002-0375-1286

\section{Martha Sabrina Barbosa Barreto}

Graduanda em Fisioterapia, Centro Universitário Estácio de Sergipe, Aracaju, Brasil.

ORCID: 0000-0002-7379-8784

\section{Ingridy Isabelle Santos}

Graduada em Enfermagem, Graduanda em Estética e Cosmética, Centro Universitário Estácio de Sergipe, Aracaju, Brasil. ORCID: 0000-0002-7956-5164

\section{Luana Renyelle de Oliveira Menezes}

Professora no Centro Universitário Estácio de Sergipe, Graduada em Estética e Cosmética, Mestre em Saúde e Ambiente, Universidade Tiradentes, Aracaju, Brasil.

ORCID: 0000-0003-0563-1875

\section{Raphaella Ingrid Santana Oliveira}

Orientadora/Professora no Centro Universitário Estácio de Sergipe, Graduada em Biomedicina, Mestre em Biotecnologia Industrial, Universidade Tiradentes, Aracaju, Brasil.

ORCID: 0000-0002-7027-9049

\section{INTRODUÇÃO}

A Toxina Botulínica (TB) é uma neurotoxina produzida por uma bactéria anaeróbica Gram positiva, chamada Clostridium botulinum. Este organismo apresenta sete tipos sorológicos (A-G), sendo o tipo A o mais potente, específica e de efeito prolongado1. A utilização da Toxina Botulínica tipo A (TBA) ocorre por conta do seu rápido crescimento em cultura e cristalização em uma forma estável, permitindo a purificação de forma eficaz, assim proporcionando maior duração dos efeitos terapêuticos ${ }^{2}$.

De acordo com a Sociedade Internacional de Cirurgia Plástica3, o Brasil ocupa o $2^{\circ}$ lugar no ranking de procedimentos estéticos não cirúrgicos realizados em todos os países, indicando, desde 2018, o uso da TB como o tratamento estético que mais cresceu no mundo. A TB, tema central deste trabalho, é utilizada para o tratamento de rugas e, através da sua aplicação, é possível analisar os efeitos em busca do rejuvenescimento ${ }^{1}$.

Desta forma, com o crescimento notável pela procura deste procedimento devido ao processo de envelhecimento, natural e precoce, que causa a formação de rugas e linhas de expressão, se faz necessário compreender o seu fundamento visando a sua indicação, contraindicação e prevenção. Assim, com relação à etiologia, as rugas são desencadeadas pela perda de colágeno e pelo aumento das atividades dos músculos $^{4}$. A sua formação apresenta dois estágios, o primeiro deles é quando ele aparece com movimento, chamada de ruga dinâmica e, quando ela fica visível, sem a necessidade de expressão facial, é chamada de ruga estática ${ }^{5,6}$. De tal modo, o uso da TB para o tratamento das rugas dinâmicas tem se tornado alvo nos procedimentos estéticos ${ }^{1}$. A atuação do tratamento da TB inicia quando ela é injetada na área facial onde estão as respectivas rugas e, como bloqueador neuromuscular, vai inibir a contração do músculo no local diante do impedimento de transmissóes de impulsos nervosos através dos neurônios ${ }^{7}$.

Analisando o efeito da toxina, é possível verificar a sua eficiência no desaparecimento temporário das rugas dinâmicas ${ }^{8}$. A recuperação deste efeito ocorre pelo surgimento de terminais nervosos e formação de novas fendas sinápticas responsáveis pela contração muscular'. Vale ressaltar que a sua aplicação, por não ser um ato cirúrgico, é uma das técnicas não invasivas mais relevantes dos tempos atuais ${ }^{4}$. Logo, juntamente com o estudo desta técnica de tratamento, seu uso se torna efetivo para promover a autoestima e melhorar a qualidade de vida dos seus pacientes ${ }^{10}$.

Diante do reconhecimento do efeito da TBA, vantagem da técnica não invasiva e a grande procura para a realização deste procedimento, questiona-se quanto à forma em que o tratamento auxilia no rejuvenescimen- 
to, eficiência e à segurança de sua aplicação. Assim, o trabalho teve como objetivo principal descrever e citar as contribuiçóes dos autores com uma abordagem exploratória e descritiva. Bem como, compreender a ação da TBA, e destacar a área da biomedicina estética para tal procedimento, o seu avanço e funcionalidade na vida das pessoas.

\section{MÉTODO}

O trabalho foi desenvolvido sob o método de caráter exploratório, adotando a pesquisa bibliográfica como seu principal aliado, baseando em análises de livros, artigos científicos, teses, dissertações acadêmicas e sites que contribuíram para o engrandecimento do respectivo artigo.

Tratando de um estudo de Revisão de Literatura e análise através de gráficos elaborados, a metodologia permitiu descrever e citar as contribuições dos autores quanto ao assunto estudado. Desta forma, sua abordagem foi considerada exploratória e descritiva; exploratória diante da possibilidade de aumentar o conhecimento em torno da Toxina Botulínica e descritiva por pretender descrever com precisão os fatos e fenômenos do seu uso no tratamento de rugas.

A busca pelos artigos científicos para o desenvolvimento do trabalho ocorreu no período de março a abril de 2020 . Os procedimentos técnicos para a pesquisa foram realizados através dos bancos de dados SciELO, PubMed, Science Direct, MedLine e Bireme, utilizando-se os seguintes descritores em português e inglês para a busca em base nacional e internacional: Toxina Botulínica (Botulinum Toxin), Envelhecimento (Aging), Botox e Rugas (Wrinkles). Diante disso, foi possível realizar uma comparação entre os bancos de dados com a quantidade de artigos publicados nos últimos 5 (cinco) anos. A análise da quantidade de artigos publicados referentes à “Toxina Botulínica”, termo em português, por cada país filiado ao banco de dados Bireme também foi passível de verificação.

$\mathrm{Na}$ busca de artigos relacionados para a discussão sobre o tema, foram escolhidos textos publicados nos anos de 2015 a 2020, sendo eles da língua portuguesa, espanhola e inglesa. Após a leitura seletiva foi feita a leitu- ra analítica para posterior interpretação dos dados mais relevantes, organizando o assunto de forma lógica para a elaboração do texto. A avaliação dos dados foi descrita através de gráficos construídos no programa GraphPad $\mathrm{PRISM}^{\circ}$ versão 5.00 e quadro com as seguintes informações: Autores, Título, Ano, Base de Dados, Objetivo e Conclusão.

\section{RESULTADO}

No desenvolvimento deste trabalho foi realizado uma busca de artigos científicos para servir como instrumento de análise sobre a aplicação da Toxina Botulínica nos procedimentos estéticos. Na busca preliminar foram pesquisados em português os descritores "Toxina Botulínica" (TB) e "Botox" (BTX) (Figura 1), sendo eles comparados entre si através de variados bancos de dados, tendo como critério os últimos cinco anos de publicações destes artigos.

Como pode ser visto, conforme o nome popular conhecido, o descritor "Botox" (BTX) teve em torno de 5.232 de artigos comparado ao descritor "Toxina Botulínica” (TB) nas bases de dados. Sugerindo que este resultado se deve por conta da constante frequência utilizada deste termo em todo o mundo, talvez por ser mais conhecido em diversas línguas e de fácil acesso nas buscas dos artigos.

Diante do crescimento dos procedimentos estéticos, foram observadas as origens dos artigos científicos através do critério quantitativo dos artigos publicados pelos países, tirando como análise os seus interesses e a busca de avanços do respectivo tema, ficando o Brasil em $5^{\circ}$ lugar nos últimos 5 anos, de acordo com o descritor em português, "Toxina Botulínica" (TB), no banco de dados Bireme (Figura 2).

\section{DISCUSSÃO}

Verifica-se que a posição do Brasil no ranking de elaboração de artigos do respectivo tema não acompanha a sua posição de $2^{\circ}$ lugar no ranking de realização de procedimentos estéticos não cirúrgicos realizados em todos os países, de acordo com Sociedade Internacional de Cirurgia Plástica ${ }^{3}$. Com isso, esses resultados demonstram e sugerem a necessidade de mais artigos científicos e estudos na área no território brasileiro a fim de alcançar o privilégio no conhecimento desta técnica, bem como o avanço da ciência estética no Brasil. De acordo com Silva ${ }^{11}$, a biomedicina estética é um ramo de grande ascensão diária onde as novidades são cons-

\section{Figura 1 - Pesquisa de artigos com descritores "Toxina Botulínica" (TB) e "Botox" (BTX).}

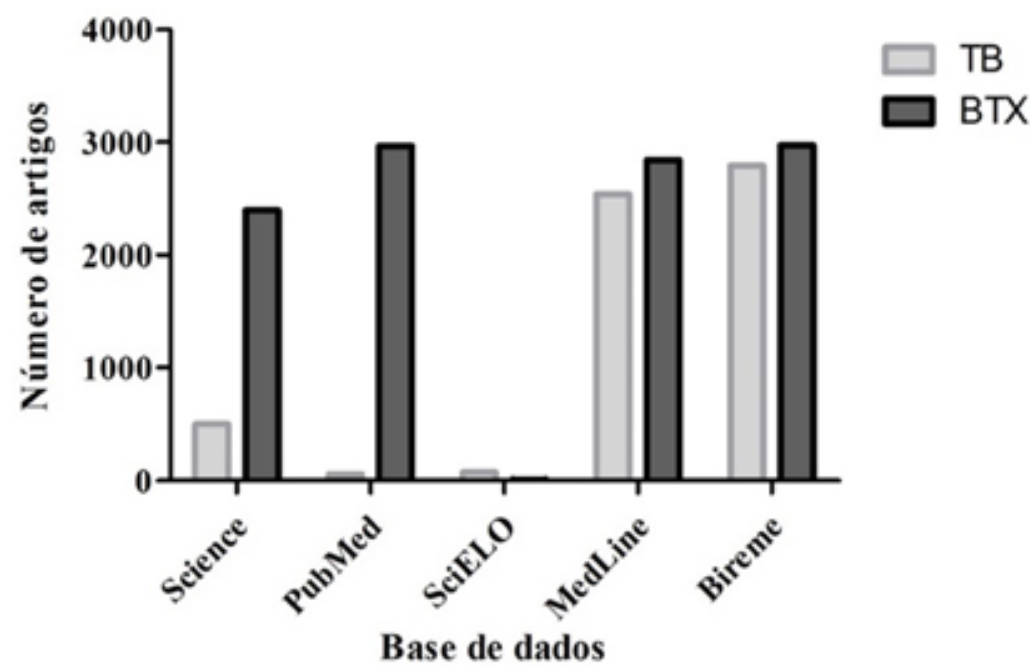

Fonte: Autoria própria (2020). 
Figura 2 - Artigos publicados por país de afiliação no site Bireme com o descritor "Toxina Botulínica" (TB).

\section{BIREME - TB}



País de Afiliação tantes, e com isso, através do estudo, seja possível desmitificar a profissão e o uso da TBA, tornando-lhe um procedimento estético melhor esclarecido na sociedade.

Desta forma, foi possível realizar análises de artigos, estabelecendo critérios de exclusão, retirando as publicações em forma de resumo, obtendo para este trabalho a utilização de artigos científicos e dissertações também com os descritores "Envelhecimento" e "Rugas", que serviram de base para o estudo, sendo os artigos incluídos no Quadro 1 a seguir:

Com a análise descritiva das referências, foi visto que o trabalho de Gart e Gutowski ${ }^{9}$ avalia, de modo geral, o uso da TBA nos procedimentos estéticos, analisando os locais apropriados para aplicação na região da face - linhas glabelares, testa, pés de galinha e linhas dos lábios periorais - e, por fim, considerando que cada paciente deve ser analisado individualmente no que tange a seleção do produto, dosagens e locais para aplicação. Ratificando as afirmações, França et al. ${ }^{13}$ contam a história da TBA e a sua evolução no decorrer dos anos, a qual foi possível descrever cada fase da toxina, considerando os benefícios que lhe foram atribuídos através de procedimentos estéticos, sugerindo assim, a substituição de procedimentos cirúrgicos para a aplicação da TBA no tratamento de rugas, sendo ele um procedimento não inva-

Fonte: Autoria própria (2020).

Quadro 1. Assuntos abordados, datas de divulgação do conteúdo digital e mídias sociais utilizadas para divulgação na atividade de extensão virtual "Estilo de vida saudável: hipertensão e diabetes", Trindade, Goiás, Brasil, 2020.

\begin{tabular}{|c|c|c|c|c|c|}
\hline AUTORES & TÍTULO & ANO & $\begin{array}{l}\text { BASE DE } \\
\text { DADOS }\end{array}$ & OBJETIVO & CONCLUSÃO \\
\hline $\begin{array}{l}\text { GART, M.S; GU- } \\
\text { TOWSKI, K.A9 }\end{array}$ & $\begin{array}{l}\text { Visão geral das Toxinas } \\
\text { Botulínicas para usos } \\
\text { estéticos/ Overview of } \\
\text { Botulinum Toxins for } \\
\text { Aesthetic Uses }\end{array}$ & 2016 & PUBMED & $\begin{array}{l}\text { Enfoca no uso estético da } \\
\text { Toxina botulínica injetável } \\
\text { analisando os locais mais } \\
\text { comuns para aplicação. }\end{array}$ & $\begin{array}{l}\text { A anatomia do paciente, comporta- } \\
\text { mentos e os padrões de injeção podem } \\
\text { ter impacto sobre a dosagem da Toxina } \\
\text { Botulínica. Assim, cada paciente deve } \\
\text { ser analisado individualmente para } \\
\text { selecionar o produto, o tratamento da } \\
\text { área e a dosagem apropriada. }\end{array}$ \\
\hline $\begin{array}{l}\text { ARANGO, } \\
\text { A.C.M.; } \\
\text { MUNOZ, S.V.F.; } \\
\text { SANDAMENTE, } \\
\text { G12 }\end{array}$ & $\begin{array}{l}\text { Mecanismo de } \\
\text { envelhecimento da } \\
\text { pele/ Mechanisms of } \\
\text { skin aging }\end{array}$ & 2017 & SCIELO & $\begin{array}{l}\text { Tratar do mecanismo } \\
\text { do envelhecimento da } \\
\text { pele de acordo com os } \\
\text { fatores intrínsecos e } \\
\text { extrínsecos. Analisar as } \\
\text { novas perspectivas de } \\
\text { prevenção e tratamento do } \\
\text { envelhecimento da pele. }\end{array}$ & $\begin{array}{c}\text { O envelhecimento da pele é um } \\
\text { processo natural em quais diferem } \\
\text { vários mecanismos para retardar o seu } \\
\text { processo. }\end{array}$ \\
\hline
\end{tabular}




\section{artigo}

Nascimento, C.G.; Taveres, M.G.V.; Nunes, M.S.; Alves, L.L.; Barreto, M.S.B.; Santos, I.I.; Menezes, L.R.O.; Oliveira, R.I.S

O uso de toxina botulínica no tratamento de rugas dinâmicas

\begin{tabular}{|c|c|c|c|c|c|}
\hline $\begin{array}{c}\text { FRANÇA et } \\
\text { al.13 }\end{array}$ & $\begin{array}{l}\text { A história da Toxina } \\
\text { Botulínica: do veneno à } \\
\text { beleza/ The history of } \\
\text { Botulinum toxin: from } \\
\text { poison to beauty }\end{array}$ & 2017 & PUBMED & $\begin{array}{l}\text { Descrever sobre o avan- } \\
\text { ço da Toxina Botulínica, } \\
\text { conhecida pelo poder be- } \\
\text { néfico nos procedimentos } \\
\text { estéticos. }\end{array}$ & $\begin{array}{l}\text { A TB é uma grande descoberta para } \\
\text { o rejuvenescimento facial, sendo um } \\
\text { procedimento menos invasivo, mais } \\
\text { fácil de realizar e de imediato resultado. }\end{array}$ \\
\hline $\begin{array}{l}\text { SATRIYASA, } \\
\text { B.K14 }\end{array}$ & $\begin{array}{l}\text { Toxina botulínica } \\
\text { (Botox) A para reduzir o } \\
\text { aparecimento de rugas } \\
\text { faciais: uma revisão da } \\
\text { literatura de uso clínico } \\
\text { e aspecto farmacoló- } \\
\text { gico/ Botulinium toxin } \\
\text { (Botox) A for reducing } \\
\text { the appearance of facial } \\
\text { wrinkles: a literature } \\
\text { review of clinical use } \\
\text { and pharmacological } \\
\text { aspect }\end{array}$ & 2019 & PUBMED & $\begin{array}{l}\text { Analisar a questão geral do } \\
\text { Botox como um tratamento } \\
\text { para a redução das rugas } \\
\text { faciais. }\end{array}$ & $\begin{array}{c}\text { Botox é um bom medicamento e } \\
\text { seguro na redução das rugas faciais. } \\
\text { Existem várias questões relacionadas } \\
\text { aos efeitos colaterais e complicações } \\
\text { após injeção. No entanto, existem } \\
\text { várias técnicas para reduzir o efeito } \\
\text { colateral e taxa de complicações após } \\
\text { a injeção. }\end{array}$ \\
\hline $\begin{array}{c}\text { BRENNAN, A; } \\
\text { HICKEY, M.8 }\end{array}$ & $\begin{array}{l}\text { Toxina Botulínica na } \\
\text { saúde da mulher: uma } \\
\text { atualização/ Botulinium } \\
\text { toxin in women's health: } \\
\text { an update }\end{array}$ & 2019 & PUBMED & $\begin{array}{l}\text { Descrever as três aplicações } \\
\text { clínicas comuns da Toxina } \\
\text { botulínica relevantes para a } \\
\text { saúde da mulher. }\end{array}$ & $\begin{array}{l}\text { O tratamento é eficaz, sendo neces- } \\
\text { sário identificar os fatores individuais } \\
\text { de cada paciente para que possa } \\
\text { influenciar a eficácia e a predisposição } \\
\text { para efeitos adversos. }\end{array}$ \\
\hline
\end{tabular}

sivo e de resultado imediato.

Contudo, preliminarmente, é necessário o estudo do envelhecimento da pele, no qual o artigo de Arango et al. ${ }^{12}$ trouxe os fatores intrínsecos - fatores fisiológicos e predisposição genética - bem como os extrínsecos como os fatores ambientais - fumo, consumo de álcool, hábitos alimentares, radiação ultravioleta, entre outros - que podem desencadear o surgimento das rugas dinâmicas, surgindo através de linhas que se formam pela contração muscular. Por meio disso, Arango et al. ${ }^{12}$ buscaram os tipos de prevenção eficazes para o retardamento do envelhecimento da pele, através da ingestão ou aplicação de produtos naturais, além do uso de filtro solar para a proteção da radiação.

A fim de prevenção e tratamento para o rejuvenescimento da pele, Satriyasa ${ }^{14}$ apontou uma análise clínica e farmacológica da TBA na redução das rugas, reafirmando a ideia do Botox como o melhor procedimento para este fim, apresentando mínimos efeitos colaterais e complicações após o seu uso. Brennan e Hicckey ${ }^{8}$ também relataram a TBA como um agente significante na saúde das mulheres, além de desempenhar uma função terapêutica no manejo dos sintomas da bexiga hiperativa, na enxaqueca crônica e nas rugas faciais, avaliando os poucos estudos encontrados na elaboração do tema e a consequente consciência de que novos estudos precisam ser feitos para o avanço do tratamento através da TBA.

Diante disso, com artigos complementares, a obra de Nogueira ${ }^{6}$ reforça a utilização da TBA para o tratamento do rejuvenescimento facial, como sendo um recurso satisfatório para os pacientes submetidos a este procedimento, demonstrando que cada vez mais, através do seu artigo, a evolução se torna acentuada no uso do Botox. Conjuntamente, apostando nos mesmos ideais, o artigo de Chaves e Paula ${ }^{15}$ também enriquece na elaboração do trabalho ao citar as contraindicações no uso de pessoas que apresentam hipersensibilidade a algum componente da TBA, na aplicação em locais infeccionados, entre outros, no intuito de assegurar um bom resultado do procedimento.

Fortalecendo a concepção das obras anteriores, Morais et al. ${ }^{5}$ fizeram uma análise quantitativa do tratamento dos pacientes submetidos a TBA na redução das rugas dinâmicas em clínica de biomedicina estética, e tiveram como resposta a procura constante pelo BTX, sendo as mulheres as principais pacientes deste procedimento estético. Neste estudo também foi possível ver a popularidade do procedimento estético por ser relativamente não invasiva, de poucos efeitos adversos, pouca gravidade e acessível quando comparada a outros procedimentos 5 .

Entretanto, Yannakopolou ${ }^{16}$ indica efeitos contrários associados no uso terapêutico e no uso cosmético da TBA, no qual é mais corriqueiro o surgimento de efeitos adversos no uso terapêutico, como a disfagia, comprometimento respiratório, fraqueza muscular generalizada e entre outros. Também Gorgojo e Rodriguez ${ }^{17}$ indicaram os erros que devem ser evitados no tratamento das rugas com TBA para que não ocorram resultados indesejados, tais como aplicaçôes com elevadas doses ou pouco tempo de intervalo de uma aplicação para outra.

Estas manifestações indesejáveis também podem ocorrer por falta de cuidado pós-tratamento por parte dos pacientes em relação as orientações recomendadas pelo profissional, como evitar esforços físicos e não abaixar a cabeça ${ }^{15}$. Ademais, Jia et al. ${ }^{18}$, através da sua meta-análise, detectaram as incidências dos eventos adversos e complicações no tratamento das rugas faciais, como dor, distúrbio ocular, ptose palpebral e pálpebras pesadas, porém confirmando a segurança na aplicação do BTX conforme o cumprimento dos padrões técnicos para utilização da droga neurotoxina.

Diante disso, conclui-se com a reflexão de Silva e Brito ${ }^{17}$ sobre a importância da interdis- 
ciplinaridade na estética e seu avanço para amenizar o processo de envelhecimento, colocando em questão a área estética como uma área de prevenção de outros problemas de saúde, bem como a melhora na qualidade de vida dos pacientes submetidos a estes tratamentos. Logo, Silva ${ }^{11}$ faz um apanhado sobre uma das áreas mais importantes para a estética na aplicação da TBA: a biomedicina estética, que por sua vez deve preparar profissionais altamente habilitados para tal procedimento seguindo protocolos, normas e indicações para obter um bom resultado no tratamento como um todo.

Assim, através da junção das referências bibliográficas com seus temas relacionados à Toxina Botulínica tipo A no tratamento das rugas dinâmicas, foi possível estabelecer elos que certifiquem a eficácia deste procedimento estético, conforme o estudo do uso, indicações, contraindicações e prevenções que devem ser feitas na aplicação da TBA. Assim, as novidades e avanços estão constantes e tornaram a área biomédica como instrumento de realizações dos desejos dos pacientes que almejam o bem estar e a autoestima através dos procedimentos de antienvelhecimento.

\section{CONCLUSÃO}

O artigo descreveu o uso da Toxina Botulínica tipo A como método para o tratamento de rugas e alcance do rejuvenescimento facial. Desta forma, foi possível verificar, pelos estudos e relatos, a eficácia da TBA nas rugas dinâmicas, resultantes de repetidos movimentos dos músculos faciais, conforme o envelhecimento cutâneo e sua relação com o mecanismo e efeito da toxina sobre as rugas.

Assim, o Botox é considerado uma das técnicas mais procuradas pelas pessoas que almejam alcançar a juventude cutânea, pois a sua aplicação minimamente invasiva apresenta raros efeitos adversos e pouca gravidade. Vale ressaltar a presença de uma análise conjunta do procedimento estético, a qual foi avaliada as normas, indicações e precauções que devem ser seguidas para a obtenção de bons resultados no tratamento.

Assim, através da elaboração deste artigo, o avanço do estudo da Toxina Botulínica tipo A apresenta uma crescente ascensão nas buscas dos artigos científicos conforme análises obtidas anteriormente pelos gráficos, situando a quantidade de estudos já elaborados do respectivo tema. Contudo, se fazem necessários mais estudos aprofundados sobre a aplicação e efeitos da TBA na redução das rugas dinâmicas juntamente com o papel da biomedicina nesta área, pois o Brasil como um dos principais países que realizam procedimentos estéticos, precisa acompanhar o ritmo científico voltado para saúde e bem-estar deste procedimento que já foi averiguado a sua efetividade.

\section{REFERÊNCIAS}

1. Uebel MR. Uso da toxina botulínica na prevenção de rugas dinâmicas: uma revisão de literatura. Lajeado. Monografia [Pós-graduação em Farmácia Estética] - Universidade do Vale do Taquari; 2019.

2. Correa GKAS, Pereira IEA, Costa JS, Lorraine J, Dias NM, Silva RIE, et al. Utilização da Toxina Botulínica tipo A para fins terapêuticos. Brazilian journal of surgery and clinical research 2019; 2(3):61-65.

3. International Society of Aesthetic Plastic Surgery - ISAPS [https:// www.isaps.org/wp-content/uploads/2019/12/ISAPS-Global-Survey-2018-Press-Release-Portuguese.pdf]. ISAPS Global Statistics [acesso em 30 abr 2020]. Disponível em: https://www.isaps.org

4. Fujita RLR, Hurtado CCN. Aspectos relevantes do uso da Toxina Botulínica no tratamento estético e seus diversos mecanismos de ação. Revista Saber Científico 2019; 8(1):120-33.

5. Morais AM, Santos IR, Schifino LNC. Análise quantitativa do tratamento de pacientes com rugas dinâmicas faciais utilizando Toxina Botulínica tipo "A" em uma clínica de biomedicina estética em Vitória da Conquista-Bahia. Revista Educação, meio ambiente e saúde 2019; 9(4):87-103.

6. Nogueira CLC. A aplicação da Toxina Botulínica tipo A no tratamento dos sinais de envelhecimento cutâneo facial. Recife. Monografia [Especialização em Biomedicina Estética]. Instituto Nacional de Ensino Superior e Pesquisa - Centro de Capacitação Educacional; 2016.

7. Mosconi PM, Oliveira RCG. Estudo da Toxina Botulínica e sua diluição. Revista Uningá 2018; 55(3):84-95.

8. Brennan A, Hickey M. Botulinium toxin in women's health: an update. Maturitas 2019; 119:21-12.

9. Gart MS, Gutowski KA. Overview of Botulinum Toxins for Aesthetic
Uses. Clinics in Plastic Surgery, 2016; 43(3):459-71.

10. Silva O, Brito JQA. O avanço da estética no processo de envelhecimento: uma revisão de literatura. Id online revista multidisciplinar e psicologia 2017; 11(35):424-40.

11. Silva ALF. Toxina botulínica na biomedicina estética: uma revisão integrativa. Rio Grande do Norte. Monografia [Graduação em biomedicina] - Faculdade de enfermagem Nova Esperança de Mossoró; 2018.

12. Arango ACM, Munoz SVF, Sandamente G. Mechanisms of skin aging. Latreia 2017; 30(2):160-70.

13. França K, Kumar AB, Fioranelli M, Lotti T, Tirant M, Roccia MG. The history of Botulinum toxin: from poison to beauty. Wiener Medizinische Wochenschrift 2017; 167:46-48.

14. Satriyasa BK. Botuliniumtoxin (Botox) A for reducing the appearance of facial wrinkles: a literature review of clinical use and pharmacological aspect. Clin Cosmet Investig Dermatol 2019; 12:223-28.

15. Chaves CTM, Paula FR. A utilização da Toxina Botulínica tipo A no rejuvenescimento. In: Anais dos $14^{\circ}$ Simpósio de TCC e $7^{\circ}$ Seminário de IC da Faculdade ICESP; 2018; Brasilia, Brasil. p. 241-251.

16. Yiannakopoulou, E. Serious and long-term adverse events associated with the therapeutic and cosmetic use of Botulinum Toxin. Pharmacology 2015; 95:65-69.

17. Gorgojo, A. M; Rodriguez R.R. Diez errores a evitar en la inyección de Toxina Botulínica. Actas Dermo Siliograficas 2015; 106(6):458-64.

18. Jia Z, Lu H, Yang X, Jin X, Wu R, Zhao J, et al. Adverse events of botulinum toxin type $A$ in facial rejuvenation: $A$ systematic Review and meta-analysis. Aesthetic Plastic Surgery 2016; 40(5):769-77. 